\title{
AN EXAMPLE OF LOW MACH (FROUDE) NUMBER EFFECTS FOR COMPRESSIBLE FLOWS WITH NONCONSTANT DENSITY (HEIGHT) LIMIT
}

\author{
Didier Bresch ${ }^{1}$, Marguerite Gisclon ${ }^{2}$ And Chi-Kun Lin ${ }^{3}$
}

\begin{abstract}
The purpose of this work is to study an example of low Mach (Froude) number limit of compressible flows when the initial density (height) is almost equal to a function depending on $x$. This allows us to connect the viscous shallow water equation and the viscous lake equations. More precisely, we study this asymptotic with well prepared data in a periodic domain looking at the influence of the variability of the depth. The result concerns weak solutions. In a second part, we discuss the general low Mach number limit for standard compressible flows given in P.-L. Lions' book that means with constant viscosity coefficients.
\end{abstract}

Mathematics Subject Classification. 35Q30.

Plenary lecture, Low Mach Number Flows Conference, June 21-25, 2004, Porquerolles, France.

\section{INTRODUCTION}

This paper is devoted to the study of the so-called low Mach number limit of compressible flows where the Mach number is given as the ratio between the characteristic velocity of the flow and the sound velocity. From a Mathematical point of view, this is the same that to study the low Froude number limit of geophysical flows: for instance shallow water flows. The Froude number being the ratio between the characteristic velocity of the flow and the gravity. In [11], the authors have studied the motion of an incompressible fluid confined to a shallow basin with a varying bottom topography. They introduce appropriate scalings into a three dimensional anisotropic eddy viscosity model to derive a two dimensional viscous lake model. Existence of global weak solutions and existence of global strong solution are proved using the dissipation property coming from the eddy viscosity. For this, they assume the bottom topography to be strictly positive. In [2], the motion of a viscous shallow water model which is of compressible type is studied. The special structure of the diffusion is also used to get an existence result of global weak solutions. The depth is now an unknown which is able to vanish. They also study the convergence to the quasi-geostrophic equation when the total depth asymptotically tends to a constant with the Rossby and the Froude number tending to 0 . The Rossby number being given by

Keywords and phrases. Compressible flows, Navier-Stokes equations, low Mach (Froude) Number limit shallow-water equations, lake equations, nonconstant density.

1 LMC-IMAG, (CNRS, INPG, UJF, INRIA) 38051 Grenoble Cedex, France. didier.bresch@imag.fr

${ }^{2}$ Université de Savoie, LAMA, UMR CNRS 5127, 73376 Le Bourget-du-lac, France. marguerite.gisclon@univ-savoie.fr

3 Department of Mathematics, National Cheng Kung University, Tianan 701 Taiwan. cklin@mail.ncku.edu.tw 
Ro $=U / \omega \ell$ where $U$ is the characteristic velocity, $\ell$ is the characteristic length and $\omega$ is the frequency of the rotation of the earth.

Here the Rossby number is fixed and we perform the asymptotic when the Froude number goes to 0 assuming the total depth converging to a function depending on $x$. Using this asymptotic, we obtain the viscous lake equations from the viscous compressible shallow water. This corresponds to the low Mach number limit for compressible flows with nonconstant density limit $b(x)$ but now with a viscosity depending on this limit density $b(x)$. All the previous works (see $[1,3,4,6-8,10]$ for complete references) concern the case where the initial density is $\rho_{0}=c+\varepsilon \widetilde{\rho}_{0}$ where $\varepsilon$ is the Mach number and $c$ is a constant. In that way the paper will be divided in two parts. In the first one, we will justify the asymptotic between the viscous shallow water equations and the lake equations. This will give an answer of a question given in [17]. Note that in the shallow water case, the model is degenerate since the viscosity depends on the height and it may vanish for weak solutions. Therefore we have to get some informations on the gradient of $h$. In the second part, we will explain the low Mach number limit associated to the standard compressible Navier-Stokes equations namely with a fixed viscosity coefficient. This will concern strong solutions. The details will be given in a forthcoming paper.

Shallow water and lake equations. In the first part of the paper, we consider the flow in a two-dimensional periodic domain $\Omega=T^{2}$. The viscous lake model reads

$$
\left\{\begin{array}{l}
\partial_{t} u^{0}+\left(u^{0} \cdot \nabla\right) u^{0}+\nabla \pi=2 \nu b^{-1} \operatorname{div}\left(b D\left(u^{0}\right)\right)-r_{0} b^{-1} u^{0}-r_{1}\left|u^{0}\right| u^{0}, \\
\operatorname{div}\left(b u^{0}\right)=0,
\end{array}\right.
$$

supplemented by the initial condition

where $D_{i j}(u)=\left(\partial_{i} u_{j}+\partial_{j} u_{i}\right) / 2$.

$$
\left.u^{0}\right|_{t=0}=u_{0}^{0}
$$

The viscous shallow water equations read

$$
\left\{\begin{array}{l}
\partial_{t}(h u)+\operatorname{div}(h u \otimes u)+\frac{h \nabla(h-b)}{\operatorname{Fr}^{2}}=2 \nu \operatorname{div}(h D(u))-r_{0} u-r_{1} h|u| u \\
\partial_{t} h+\operatorname{div}(h u)=0
\end{array}\right.
$$

where $\mathrm{Fr}=\sqrt{U \ell} / g=\varepsilon>0$ denotes the Froude number, $U$ being the characteristic velocity, $\ell$ the characteristic depth and $g$ the gravity. System (1.1) is supplemented with initial conditions

$$
\left.h\right|_{t=0}=h_{0},\left.\quad(h u)\right|_{t=0}=m_{0}
$$

where $h_{0}$ is assumed to be positive. This model is formally derived from the three-dimensional Navier-Stokes equations with free surface, where the normal stress is determined from the air pressure and no capillarity effects, see [9]. The drag terms $r_{0} u\left(r_{0}>0\right)$ in the laminar case and $r_{1} h|u| u\left(r_{1}>0\right)$ in the turbulent regime are obtained from the friction condition on the bottom, see [18].

In the sequel, we assume $f=0$ without loss of generality since all the analysis can be extended to the case of regular enough $f$. We will also consider the gradient operator $\nabla u^{0}$ instead of $D(u)$ since the study is the same.

\section{Existence RESUlts}

Before investigating the case of vanishing Froude or Mach number, we recall the existence results of the systems obtained respectively in $[2,11]$.

The viscous shallow water equations. The energy inequality associated to System (1.2) reads as

$\frac{1}{2} \int_{\Omega}\left(h|u|^{2}+\left|\frac{h-b}{\mathrm{Fr}}\right|^{2}\right)+\nu \int_{0}^{T} \int_{\Omega} h|D(u)|^{2}+r_{0} \int_{0}^{T} \int_{\Omega}|u|^{2}+r_{1} \int_{0}^{T} \int_{\Omega} h|u|^{3} \leq \frac{1}{2} \int_{\Omega}\left(h_{0}\left|u_{0}\right|^{2}+\left|\frac{h_{0}-b}{\operatorname{Fr}}\right|^{2}\right)$. 
The initial data are taken in such way that

$$
\begin{gathered}
h_{0} \in L^{2}(\Omega), \quad \frac{\left|m_{0}\right|^{2}}{h_{0}} \in L^{1}(\Omega), \\
\nabla \sqrt{h_{0}} \in\left(L^{2}(\Omega)\right)^{2}, \quad-r_{0} \log _{-} h_{0} \in L^{1}(\Omega)
\end{gathered}
$$

where $\log _{-} g=\log \min (g, 1)$. The initial height is assumed to be positive and the function $b(x)$ such that $b(x) \geq 0$.

We say that $(h, u)$ is a weak solution on $(0, T)$ of Shallow-water equations if the System (1.2) holds in $\left(\mathcal{D}^{\prime}((0, T) \times \Omega)\right)^{3}$ with $h \geq 0$ a.e., the initial data are satisfied in $\mathcal{D}^{\prime}(\Omega)$ and the energy inequality is satisfied for a.e. non negative $t$. The following regularity properties are satisfied:

$$
\begin{gathered}
\nabla \sqrt{h} \in L^{\infty}\left(0, T ;\left(L^{2}(\Omega)\right)^{2}\right), \quad \sqrt{h} u \in L^{\infty}\left(0, T ;\left(L^{2}(\Omega)\right)^{2}\right), \\
\sqrt{h} D(u) \in\left(L^{2}\left(0, T ;\left(L^{2}(\Omega)\right)\right)^{4}, \quad \nabla h \in\left(L^{2}\left(0, T ;\left(L^{2}(\Omega)\right)\right)\right)^{2},\right. \\
\sqrt{r}_{0} u \in\left(L^{2}\left(0, T ;\left(L^{2}(\Omega)\right)\right)\right)^{4}, \quad r_{1}^{1 / 3} h^{1 / 3} u \in\left(L^{3}\left(0, T ;\left(L^{3}(\Omega)\right)\right)\right)^{2} .
\end{gathered}
$$

The following existence result has been proved in [2] with $b=0$.

Theorem 2.1. Let $m_{0}, h_{0}$ satisfy (2.3) and assume $r_{0}, r_{1}>0$. Then there exists a global weak solution of (1.2).

This results remains valid even if $b \geq 0$ since the term $h^{\varepsilon} \nabla b / \mathrm{Fr}^{2}$ may be seen as an exterior force and Fr is fixed. The reader interested by the shallow water equations and its application in geophysics is referred to [18].

The viscous lake equations. The energy inequality associated to System (1.1) reads as

$$
\frac{1}{2} \int_{\Omega} b\left|u^{0}\right|^{2}+\nu \int_{0}^{T} \int_{\Omega} b\left|D\left(u^{0}\right)\right|^{2}+r_{0} \int_{0}^{T} \int_{\Omega}\left|u^{0}\right|^{2}+r_{1} \int_{0}^{T} \int_{\Omega} b\left|u^{0}\right|^{3} \leq \frac{1}{2} \int_{\Omega} b\left|u_{0}^{0}\right|^{2} .
$$

The initial data $u_{0}^{0}$ are taken in such way that

$$
\sqrt{b} u_{0}^{0} \in\left(L^{2}(\Omega)\right)^{2}
$$

where $b u_{0}^{0}=0$ on $b^{-1}(\{0\})$.

We say that $u^{0}$ is a weak solution on $(0, T)$ of lake equations if the System $(1.1)$ holds in $\left(\mathcal{D}^{\prime}((0, T) \times \Omega)\right)^{3}$ with $b \geq 0$ a.e., the initial data are satisfied in $\mathcal{D}^{\prime}(\Omega)$ and the energy inequality is satisfied for a.e. non negative $t$. The following regularity properties are satisfied:

$$
\begin{array}{cc}
\sqrt{b} u^{0} \in L^{\infty}\left(0, T ;\left(L^{2}(\Omega)\right)^{2}\right), & \sqrt{b} D\left(u^{0}\right) \in\left(L^{2}\left(0, T ;\left(L^{2}(\Omega)\right)^{4}\right),\right. \\
\sqrt{r}_{0} u^{0} \in\left(L^{2}\left(0, T ;\left(L^{2}(\Omega)\right)\right)^{2},\right. & r_{1}^{1 / 3} b^{1 / 3} u^{0} \in L^{3}\left(0, T ;\left(L^{3}(\Omega)\right)^{2}\right) .
\end{array}
$$

The following existence result may be proved completed [11].

Theorem 2.2. Let $u_{0}^{0}$ satisfies (2.4) and assume $r_{0}, r_{1}>0$. Then there exists a global weak solution $u^{0}$ of (1.1) that means a solution such that the regularity previously cited is obtained.

Under the assumption that $b$ is strictly positive, we can prove the following regularity result that will be useful for the weak-strong convergence result. 
Theorem 2.3. Let $b$ be regular enough with $b \geq c>0$. Let $u_{0}^{0}$ be such that $u_{0}^{0} \in\left(H^{3}(\Omega)\right)^{2}$. Then there exists a unique weak solution $u^{0}$ of (1.1) such that

$$
\begin{gathered}
\left.u^{0} \in L^{\infty}\left(0, T ;\left(H^{3}(\Omega)\right)^{2}\right) \cap L^{2}\left(0, T ;\left(H^{4}(\Omega)\right)^{2} \cap V\right)\right), \\
\partial_{t} u^{0} \in L^{2}\left(0, T ;\left(H^{2}(\Omega)\right)^{2}\right) \cap L^{\infty}(0, T ; V)
\end{gathered}
$$

where $V$ is the space of the function $v$ which belong to $H^{1}$ such that we have $\operatorname{div}(b v)=0$.

The reader interested by the inviscid lake equations is referred to [12], see also [17].

\section{MAin Results}

In this paper, we are interested by the low Mach (Froude) number limit for compressible Navier-Stokes equations with a nonconstant density limit.

In a first part, we give a physical example where such asymptotic occurs, mainly the asymptotic between the shallow water equations and the viscous lake equations.

We will prove the following asymptotic results in the well prepared case assuming that $b \geq c>0$.

Theorem 3.1. Let us assume that $u_{0}^{0} \in\left(H^{3}(\Omega)\right)^{2}$ and

$$
\left(u_{0}^{\varepsilon}, h_{0}^{\varepsilon}\right) \rightarrow\left(u_{0}^{0}, 0\right) \text { in }\left(L^{2}(\Omega)\right)^{3}, \quad\left(h_{0}^{\varepsilon}-b\right) / \varepsilon \rightarrow 0 \text { in } L^{2}(\Omega)
$$

as $\varepsilon$ goes to 0 . Then, denoting by $\left(h^{\varepsilon}, u^{\varepsilon}\right)$ a global weak solution of $(1.2)$, then

$$
\begin{gathered}
u^{\varepsilon} \rightarrow u^{0} \text { in } L^{\infty}\left(0, T ;\left(L^{2}(\Omega)\right)^{2}\right), \\
\left(h^{\varepsilon}-b\right) / \varepsilon \rightarrow 0 \text { in } L^{\infty}\left(0, T ; L^{2}(\Omega)\right), \quad \nabla\left(h^{\varepsilon} / b\right) \rightarrow 0 \text { in } L^{2}\left(0, T ;\left(L^{2}(\Omega)\right)^{2}\right),
\end{gathered}
$$

when $\varepsilon \rightarrow 0$, where $u^{0}$ is the global strong solution of the viscous lake equation (1.1).

In the second part, we will describe the standard compressible Navier-Stokes equations and give some comments on the asymptotic that will be performed in a forthcoming paper.

\section{Formal ASYmptotic}

Let us denote $\mathrm{Fr}=\varepsilon$. We use the following ansatz

$$
u^{\varepsilon}=u^{0}+\varepsilon u^{1}+\ldots, \quad h^{\varepsilon}=h^{0}+\varepsilon h^{1}+\ldots
$$

Denoting $\Psi(t, x)=\frac{h-b(x)}{\varepsilon}$, System 1.2 reads

$$
\left\{\begin{array}{l}
\partial_{t} u+u \cdot \nabla u+\frac{\nabla \Psi}{\varepsilon}=\frac{2}{h} \nu \operatorname{div}(h D(u))-\frac{1}{h} r_{0} u-r_{1}|u| u+f, \\
\partial_{t} \Psi+\operatorname{div}(\Psi u)+\frac{1}{\varepsilon} \operatorname{div}(b u)=0 .
\end{array}\right.
$$

Putting this ansatz in the shallow water equation, we get at order $1 / \varepsilon$ on the momentum equation and $1 / \varepsilon$ on the height equation

$$
h^{0}=b(x), \quad \operatorname{div}\left(b u^{0}(t, x)\right)=0 .
$$

Let us now look at order $\varepsilon^{0}$. We obtain

$$
\partial_{t} u^{0}+\left(u^{0} \cdot \nabla\right) u^{0}+\nabla \Psi^{1}=\frac{2}{b} \nu \operatorname{div}\left(b D\left(u^{0}\right)\right)-\frac{1}{h^{0}} r_{0} u^{0}-r_{1}\left|u^{0}\right| u^{0} .
$$

Thus, we get the viscous lake equations. 


\section{Proof of the main Result}

The convergence for well prepared data is not straightforward even if the asymptotic is obtained using a standard weak-strong energy estimate since the asymptotic solution is a little bit more regular than the initial solutions of the shallow water equations. The height $h^{\varepsilon}$ may vanish, then we have to get some informations about its gradient in order to be able to conclude.

At the beginning, we proceed, classically, by a weak-strong estimation procedure. The reader interested by similar study on various systems is referred for instance to $[2,14]$ and references cited therein. Using the energy inequality for weak solution of (1.2), the energy equality for the limit solution, the momentum equations of weak solutions (1.2) tested against $u^{\varepsilon}$, we get the following estimates:

$$
\begin{aligned}
\int_{\Omega} h^{\varepsilon} \frac{\left|u^{\varepsilon}-u^{0}\right|^{2}}{2}+\frac{1}{2} \int_{\Omega}\left|\frac{h^{\varepsilon}-b}{\varepsilon}\right|^{2}+\nu & \int_{0}^{t} \int_{\Omega} h^{\varepsilon}\left|\nabla\left(u^{\varepsilon}-u^{0}\right)\right|^{2} \\
& +\int_{0}^{t} \int_{\Omega} r_{0}\left|u^{\varepsilon}-u^{0}\right|^{2}+\int_{0}^{t} \int_{\Omega}\left(r_{1} h^{\varepsilon}\left|u^{\varepsilon}\right| u^{\varepsilon}-r_{1} h^{\varepsilon}\left|u^{0}\right| u^{0}\right)\left(u^{\varepsilon}-u^{0}\right) \leq \sum_{i=1}^{4} I_{i}
\end{aligned}
$$

where

$$
\begin{gathered}
I_{1}=\int_{\Omega} h_{0}^{\varepsilon} \frac{\left|u_{0}^{\varepsilon}-u_{0}^{0}\right|^{2}}{2}+\frac{1}{2} \int_{\Omega}\left(b-h_{0}^{\varepsilon}\right)\left|u_{0}^{0}\right|^{2}+\frac{1}{2} \int_{\Omega}\left|\frac{h_{0}^{\varepsilon}-b}{\varepsilon}\right|^{2} \\
I_{2}=\int_{0}^{t} \int_{\Omega}\left(b-h^{\varepsilon}\right) u^{\varepsilon} \partial_{t} u^{0}+\nu \int_{0}^{t} \int_{\Omega}\left(b-h^{\varepsilon}\right) \nabla u^{0} \cdot \nabla u^{\varepsilon}-\int_{0}^{t} \int_{\Omega} h^{\varepsilon} u^{\varepsilon} \cdot \nabla u^{0} u^{\varepsilon}+\int_{0}^{t} \int_{\Omega} b u^{0} \cdot \nabla u^{0} u^{\varepsilon}, \\
I_{3}=-\frac{1}{\varepsilon^{2}} \int_{0}^{t} \int_{\Omega}\left(h^{\varepsilon}-b\right) \operatorname{div}\left(\left(h^{\varepsilon}-b\right) u^{0}\right)+\int_{0}^{t} \int_{\Omega}\left(b-h^{\varepsilon}\right) r_{1}\left|u^{0}\right| u^{0} u^{\varepsilon}-\int_{0}^{t} \int_{\Omega}\left(\operatorname{div} b u^{\varepsilon}\right) \pi
\end{gathered}
$$

and

$$
I_{4}=\frac{1}{2} \int_{\Omega}\left(h^{\varepsilon}-b\right)\left|u^{0}\right|^{2}+\nu \int_{0}^{t} \int_{\Omega}\left(h^{\varepsilon}-b\right)\left|\nabla u^{0}\right|^{2}+\int_{0}^{t} \int_{\Omega}\left(h^{\varepsilon}-b\right) r_{1}\left|u^{0}\right|^{3} .
$$

We rewrite the last two nonlinear terms in $I_{2}$,

$$
\begin{array}{r}
I_{2}=\int_{0}^{t} \int_{\Omega}\left(b-h^{\varepsilon}\right) u^{\varepsilon} \partial_{t} u^{0}+\nu \int_{0}^{t} \int_{\Omega}\left(b-h^{\varepsilon}\right) \nabla u^{0} \cdot \nabla u^{\varepsilon}-\int_{0}^{t} \int_{\Omega} h^{\varepsilon}\left(u^{\varepsilon}-u^{0}\right) \cdot \nabla u^{0}\left(u^{\varepsilon}-u^{0}\right) \\
-\int_{0}^{t} \int_{\Omega} h^{\varepsilon}\left(u^{\varepsilon}-u^{0}\right) \cdot \nabla u^{0} u^{0}+\int_{0}^{t} \int_{\Omega}\left(b-h^{\varepsilon}\right) u^{0} \cdot \nabla u^{0} u^{\varepsilon} .
\end{array}
$$

We now use the momentum equation satisfied by $u^{0}$ and multiply it by $\left(b-h^{\varepsilon}\right) u^{\varepsilon}$, we can rewrite $I_{2}$ as

$$
\begin{aligned}
I_{2}=-\int_{0}^{t} \int_{\Omega} h^{\varepsilon}\left(u^{\varepsilon}-u^{0}\right) \cdot \nabla u^{0}\left(u^{\varepsilon}-u^{0}\right)-\int_{0}^{t} \int_{\Omega} h^{\varepsilon}\left(u^{\varepsilon}-u^{0}\right) \cdot \nabla u^{0} u^{0} \\
\quad+\nu \int_{0}^{t} \int_{\Omega} b \nabla\left(\frac{h^{\varepsilon}}{b}\right) \nabla u^{0} u^{\varepsilon}+\int_{0}^{t} \int_{\Omega} \pi \operatorname{div}\left(u^{\varepsilon} \frac{b-h^{\varepsilon}}{b}\right)-\frac{r_{0} u^{0}}{b}\left(b-h^{\varepsilon}\right) u^{\varepsilon}-r_{1}\left|u^{0}\right| u^{0}\left(b-h^{\varepsilon}\right) u^{\varepsilon} .
\end{aligned}
$$

Let us assume for the moment that $h^{\varepsilon} \rightarrow b$ in $L^{\infty}\left(0, T ; L^{2}(\Omega)\right), \nabla\left(h^{\varepsilon} / b\right) \rightarrow 0$ in $L^{2}\left(0, T ;\left(L^{2}(\Omega)\right)^{2}\right)$ and let us prove the theorem, letting $\varepsilon$ go to 0 in the energy estimate. We know that $I_{1}$ converges to 0 by assumptions on the data. The group $I_{4}$ converges to 0 since $h^{\varepsilon} \rightarrow b$ in $L^{2}\left(0, T ; L^{2}(\Omega)\right)$ and $u^{0}$ is smooth enough. In the group $I_{2}$, the first term is controlled by a Gronwall's type argument since $\nabla u^{0}$ is smooth enough. The second term and the fourth terms converge to 0 since $u^{\varepsilon}$ converges weakly to $v$ such that $\operatorname{div}(b v)=0, \operatorname{div}\left(h^{\varepsilon} u^{\varepsilon}\right) \rightarrow 0$ and $h^{\varepsilon} \rightarrow b$ in $L^{\infty}\left(0, T ; L^{2}(\Omega)\right)$. The last term converges since $u^{\varepsilon}$ is uniformly bounded in $L^{2}\left(0, T ;\left(L^{2}(\Omega)\right)^{2}\right)$ and $\nabla\left(h^{\varepsilon}-b\right) \rightarrow 0$ in $L^{2}\left(0, T ; L^{2}(\Omega)\right)$. Concerning the third quantity $I_{3}$, the last two terms converge to 0 
since $u^{\varepsilon}$ is uniformly bounded in $L^{2}\left(0, T ;\left(L^{2}(\Omega)\right)^{2}\right)$ and $\operatorname{div}\left(h^{\varepsilon} u^{\varepsilon}\right)$ converges to 0 . The first term is rewritten as follows:

$$
-\frac{1}{\varepsilon^{2}} \int_{0}^{t} \int_{\Omega}\left(h^{\varepsilon}-b\right) \operatorname{div}\left(\left(h^{\varepsilon}-b\right) u^{0}\right)=\frac{1}{2 \varepsilon^{2}} \int_{0}^{t} \int_{\Omega}\left(h^{\varepsilon}-b\right)^{2} u^{0} \cdot \nabla \ln b .
$$

This term is a new term compared to the study made in [2], we will control it by a Gronwall's type argument since $\left(h^{\varepsilon}-b\right) / \varepsilon$ is in the left-hand side.

Let us now prove the uniform bounds on $\nabla\left(h^{\varepsilon} / b\right) / \varepsilon$. This will be done following the calculations made in [2]. We remark that the new term in the momentum equation compared to [2] is the term $-h^{\varepsilon} \nabla b / \varepsilon^{2}$. If $\varepsilon$ is fixed this term may be seen as an external force and we obtain the same conclusion as in [2] concerning the existence result using the equalities proved in [2]. These equalities are linked to the multiplier $\nabla h^{\varepsilon} / h^{\varepsilon}$. But here, in order to pass to the limit, the estimates have to be uniform with respect to $\varepsilon$, therefore we have to adapt the test function. We will see that we are able to conclude if we use the following test function $\varphi=\nabla\left(h^{\varepsilon} / b\right) / h^{\varepsilon} / b$. The natural one $\nabla\left(h^{\varepsilon}-b\right) / h^{\varepsilon}$ seems to be not sufficient. Indeed testing the momentum equation by it gives the term

$$
\int_{\Omega} u \cdot \nabla u \nabla b
$$

to be estimate. The problem being that $\nabla u$ is not square integrable.

In the sequel, we will omit the indices $\varepsilon$ for the sake of simplicity.

Lemma 5.1. We have the following equality:

$$
\begin{aligned}
\frac{1}{2} \frac{\mathrm{d}}{\mathrm{d} t} \int_{\Omega} h\left|\nabla \ln \frac{h}{b}\right|^{2}+\int_{\Omega} h \nabla u \cdot \nabla \ln \left(\frac{h}{b}\right) & \nabla \ln \left(\frac{h}{b}\right) \\
& +\int_{\Omega} h \nabla u \cdot \nabla \ln b \nabla \ln \frac{h}{b}+\int_{\Omega} h u \cdot \nabla \nabla \ln b \nabla \ln \frac{h}{b}+\int_{\Omega} h \nabla \operatorname{div} u \nabla \ln \frac{h}{b}=0 .
\end{aligned}
$$

Proof. This lemma is obtained following the calculation done in [2], looking at the equation satisfied by $\ln h / b$, differentiating with respect to the space variable and multiplying by $h \nabla \ln (h / b)$.

More precisely, using the mass equation, we have

$$
\partial_{t}\left(\frac{h}{b}\right)+u \cdot \nabla\left(\frac{h}{b}\right)+\frac{h u \cdot \nabla b}{b^{2}}+\frac{h}{b} \operatorname{div} u=0 .
$$

Dividing by $h / b$, we get

$$
\partial_{t} \ln \left(\frac{h}{b}\right)+u \cdot \nabla \ln \frac{h}{b}+u \cdot \nabla \ln b+\operatorname{div} u=0 .
$$

Deriving this equation with respect to $x_{i}$, we obtain

$$
\partial_{t} \nabla \ln \left(\frac{h}{b}\right)+u \cdot \nabla \nabla \ln \left(\frac{h}{b}\right)+\nabla u \cdot \nabla \ln \left(\frac{h}{b}\right)+\nabla u \cdot \nabla \ln b+u \cdot \nabla \nabla \ln b+\nabla \operatorname{div} u=0 .
$$

Multiplying this equation by $h \nabla \ln (h / b)$ and using the mass equation, this gives

$$
\begin{aligned}
\frac{1}{2}\left[\partial_{t}\left(h\left|\nabla \ln \left(\frac{h}{b}\right)\right|^{2}\right)+\operatorname{div}\left(\left.h u \cdot \nabla \ln \left(\frac{h}{b}\right)\right|^{2}\right)\right] & +h \nabla u \nabla \ln \left(\frac{h}{b}\right) \nabla \ln \left(\frac{h}{b}\right) \\
& +h \nabla u \nabla \ln b \nabla \ln \left(\frac{h}{b}\right)+h u \nabla \nabla \ln b \nabla \ln \left(\frac{h}{b}\right)+h \nabla \operatorname{div} u \nabla \ln \left(\frac{h}{b}\right)=0 .
\end{aligned}
$$

Integrating with respect to the space variable, this gives the lemma. 
We can prove now the following equality which generalizes the equality derived in [2], Lem. 4 p. 220.

\section{Lemma 5.2 .}

$$
\begin{aligned}
\frac{\mathrm{d}}{\mathrm{d} t}\left(\int_{\Omega} h \mid u\right. & \left.+\left.\nu \nabla \ln \left(\frac{h}{b}\right)\right|^{2}+\left|\frac{h-b}{\varepsilon}\right|^{2}\right)-\nu r_{0} \frac{\mathrm{d}}{\mathrm{d} t} \int_{\Omega} \ln \frac{h}{b}+r_{0} \int_{\Omega}|u|^{2}+\nu \int_{\Omega} b^{2}\left|\frac{\nabla(h / b)}{\varepsilon}\right|^{2} \\
+ & \int_{\Omega} h|\nabla u|^{2}+\int_{\Omega} r_{1} h|u|^{3}=\nu \int_{\Omega} h \nabla u:{ }^{t} \nabla u-\nu \int_{\Omega} h u \cdot(\nabla \nabla \ln b) u-\nu^{2} \int_{\Omega} h u \nabla \nabla \ln b \nabla \ln \frac{h}{b} \\
& \quad \nu \int_{\Omega} \nabla b \frac{h-b}{\varepsilon} \frac{\nabla(h / b)}{\varepsilon}+r_{0} \nu \int_{\Omega} u \cdot \nabla \ln b-r_{1} \nu \int_{\Omega}|u| u \cdot \nabla h+\int_{\Omega} \nu r_{1} h|u| u \cdot \nabla \ln b .
\end{aligned}
$$

Proof. This equality is obtained multiplying the momentum equation by $\nabla \ln (h / b)$ and using the equality coming from Lemma 1.

Let us look at each term of the momentum equation tested against $\nabla \nabla(h / b)$. Using the mass equation, we deduce that

$$
\int_{\Omega} h\left(\partial_{t} u+u \cdot \nabla u\right) \cdot \nabla \ln \left(\frac{h}{b}\right)=\frac{\mathrm{d}}{\mathrm{d} t} \int_{\Omega}\left[b u \cdot \nabla\left(\frac{h}{b}\right)\right]+\int_{\Omega} b u \cdot \nabla\left[\frac{\operatorname{div}(h u)}{b}\right]+\int_{\Omega} b u \cdot \nabla u \nabla\left(\frac{h}{b}\right) .
$$

Rewriting the two last terms, this reads

$$
\int_{\Omega} h\left(\partial_{t} u+u \cdot \nabla u\right) \cdot \nabla\left(\frac{h}{b}\right) \frac{b}{h}=\frac{\mathrm{d}}{\mathrm{d} t} \int_{\Omega}\left[b u \cdot \nabla\left(\frac{h}{b}\right)\right]-\int_{\Omega} h \nabla u:{ }^{t} \nabla u+\int_{\Omega} h u \cdot \nabla(\nabla \ln b) u .
$$

Now we look at the diffusive term, we have

$$
-\nu \int_{\Omega} \operatorname{div}(h \nabla u) \nabla \ln \left(\frac{h}{b}\right)=\nu \int_{\Omega} b \nabla u: \nabla \nabla\left(\frac{h}{b}\right)-\nu \int_{\Omega} h \nabla u \cdot \nabla \ln \left(\frac{h}{b}\right) \nabla \ln \left(\frac{h}{b}\right) .
$$

Concerning now, the pressure term, we write

$$
\int_{\Omega} h \frac{\nabla(h-b)}{\varepsilon^{2}} \nabla \ln \left(\frac{h}{b}\right)=\int_{\Omega} \frac{\nabla b}{\varepsilon^{2}} \cdot \nabla\left(\frac{h}{b}\right)(h-b)+\int_{\Omega} \frac{b^{2}}{\varepsilon^{2}}\left|\nabla\left(\frac{h}{b}\right)\right|^{2}
$$

It remains now the drag terms, they give

$$
\int_{\Omega} r_{0} u \cdot \nabla \ln \left(\frac{h}{b}\right)=-r_{0} \frac{\mathrm{d}}{\mathrm{d} t} \int_{\Omega} \ln \left(\frac{h}{b}\right)-r_{0} \int_{\Omega} u \cdot \nabla \ln b
$$

and

We now remark that

$$
\int_{\Omega} r_{1} h|u| u \cdot \nabla \ln \left(\frac{h}{b}\right)=\int_{\Omega} r_{1}|u| u \nabla h-\int_{\Omega} r_{1} h|u| u \cdot \nabla \ln b
$$

$$
-\int_{\Omega} b \nabla u: \nabla \nabla \frac{h}{b}-\int_{\Omega} h \nabla \operatorname{div} u \cdot \nabla \ln \frac{h}{b}-\int_{\Omega} h \nabla u \nabla \ln b \nabla \ln \frac{h}{b}=0 .
$$

Collecting all the previous equalities and using the equality given in the preceding Lemma, we get the desired equality. 
Proof of the convergence. Let us prove that we control all the terms in the right-hand side in the equality given in Lemma 5.2. The first term is easily controlled since

$$
\int_{0}^{T}\left|J_{1}\right| \leq \int_{0}^{T} \int_{\Omega} h|\nabla u|^{2} \leq c_{1}
$$

If $b \geq c>0$ and $b \in W^{2, \infty}(\Omega)$, the second term is also controlled by

$$
\int_{0}^{T}\left|J_{2}\right| \leq \int_{0}^{T} \int_{\Omega} h|u|^{2} \leq c_{2}
$$

If $h \in W^{2, \infty}(\Omega)$ then

$$
\int_{0}^{T}\left|J_{3}\right| \leq c \varepsilon\|u\|_{L^{2}\left(Q_{T}\right)}^{2}+\frac{1}{\varepsilon}\|\nabla h\|_{L^{2}\left(Q_{T}\right)} .
$$

The fourth term is controlled as follows:

$$
\int_{0}^{T}\left|J_{4}\right| \leq\left\|\frac{h-b}{\varepsilon}\right\|_{L^{2}\left(0, T ; L^{2}(\Omega)\right)}^{2}+\frac{\nu^{2}}{2 \varepsilon^{2}}\left\|\nabla b \nabla \frac{h}{b}\right\|_{L^{2}\left(0, T ; L^{2}(\Omega)\right)}^{2} .
$$

The last terms are easily controlled using the bounds on $(h, u)$. Collecting all the informations and using strongly that $b \geq c>0$, Lemma 5.2 gives the desired convergence used in the proof of the main theorem.

Remark. The case where $b$ is assumed to vanish on the shore is an interesting difficult subject which will be discussed in a forthcoming paper. The proof used in the previous section does not seems to be easily adaptable to such configurations even in simpler cases.

\section{LOW MACH NUMBER LIMIT FOR COMPRESSIBLE FLOWS With NONCONSTANT DENSITY LIMIT}

We now look at the asymptotic when the Mach number goes to zero on the following compressible NavierStokes system

where

$$
\begin{gathered}
\partial_{t} \rho+\operatorname{div}(\rho u)=0, \\
\partial_{t}(\rho u)+\operatorname{div}(\rho u \otimes u)-\nu \Delta u-(\lambda+\nu) \lambda \nabla \operatorname{div} u+\rho \frac{\nabla \Pi(\rho, \bar{\rho})}{\varepsilon^{2}(\gamma-1)}=\rho f
\end{gathered}
$$

$$
\Pi(\rho, \bar{\rho})=\int_{\bar{\rho}}^{\rho} \frac{P(s)}{s^{2}} \mathrm{~d} s+\frac{P(\rho)}{\rho}-\frac{P(\bar{\rho})}{\bar{\rho}} .
$$

The reference density $\bar{\rho}$ depends on $x$. We first remark that systems mathematically studied during these last years assume the reference density to be equal to a constant $\bar{\rho}=1$ in the barotropic case $P(\rho)=a \rho^{\gamma}$. In this case

$$
\rho \nabla \Pi(\rho, \bar{\rho})=a \nabla \rho^{\gamma} .
$$

Remark. The Saint-Venant equations correspond to the case $\bar{\rho}=b$ and $P(\rho)=\frac{1}{2} \rho^{2}$.

Formal calculations. The energy inequality gives the following information on the density

$$
\frac{\mathrm{d}}{\mathrm{d} t} \int_{\Omega} F(\rho, \bar{\rho}) \leq 0
$$

where

$$
F\left(s_{1}, s_{2}\right)=s_{2} \int_{s_{2}}^{s_{1}} \frac{P(s)}{s^{2}} \mathrm{~d} s-\frac{P\left(s_{1}\right)}{s_{1}}\left(s_{2}-s_{1}\right) .
$$


Thus assuming that $F\left(s_{1}, s_{2}\right)$ satisfies

for some power of $\gamma$ we get that

$$
F\left(s_{1}, s_{2}\right) \geq c\left|s_{1}-s_{2}\right|^{\gamma}
$$

$$
(\rho-\bar{\rho}) / \varepsilon \text { is uniformly bounded in } L^{\infty}\left(0, T ; L^{\gamma}(\Omega)\right)
$$

and thus $\rho \rightarrow \bar{\rho}$ in $\mathcal{C}\left(0, T ; L^{\gamma}(\Omega)\right)$. Let $\varepsilon$ go to zero, then the mass equation gives

$$
\operatorname{div}\left(\bar{\rho} u^{0}\right)=0
$$

Using now the momentum equation, we get at the main order

$$
\bar{\rho}\left(\partial_{t} \bar{u}+\bar{u} \cdot \nabla \bar{u}\right)-\mu \Delta \bar{u}-(\lambda+\mu) \nabla \operatorname{div} \bar{u}+\bar{\rho} \nabla \bar{p}=\bar{\rho} f .
$$

Mathematical justification. Several asymptotic analysis concerning the zero Mach number limit of classical solutions to the compressible Euler equations for non-isentropic fluids in a domain $\Omega$ of $R^{d}$ have been performed. For $\Omega$ an exterior domain, the asymptotic has been recently mathematically proven in [1] extending the results of $[15,16]$ which concerned $\Omega=R^{d}$. The case $\Omega=T^{d}$ is completely open. Only formal results have been performed in [5] and partial answer have been made in [16]. We can say that the studies when the density converges to a nonconstant density will follow exactly the same lines and difficulties since it involves the same kind of wave equation depending on the density. For instance in the case $\Omega=T^{d}$, we can think that, in the ill prepared case, an extra term will be obtained formally in the limit of the momentum equation. Namely, we will get

$$
\bar{\rho}\left(\partial_{t} u+u \cdot \nabla u\right)-\mu \Delta u-(\lambda+\mu) \nabla \operatorname{divu}+\bar{\rho} \nabla P+\nabla q=\bar{\rho} f
$$

The term $\nabla q$ being obtained from the oscillating part of the velocity. Details will be given in a forthcoming paper.

Remark. Note that in our case, the extra term coming from the wave propagation is of the form $\bar{\rho} \nabla p_{2}+\nabla q$ as in the nonisentropic case. But here the exchange in the energy between waves and mean velocity is given through $\nabla q$ since $u$ satisfies here $\operatorname{div}(\bar{\rho} u)=0$.

Acknowledgements. D. Bresch and M. Gisclon, are partially supported by the french groupement de Recherche (GdR) "Équations d'Amplitudes et Propriétés Qualitatives" (EAPQ), managed by É. Lombardi, of the Centre National de Recherches Scientifiques (CNRS). They are also supported by a Rhône-Alpes fellowship obtained in 2004 on problems related to viscous shallow water equations and by the "ACI jeunes chercheurs 2004" du ministère de la Recherche "Études mathématiques de paramétrisations en océanographie".

\section{REFERENCES}

[1] T. Alazard, Incompressible limit of the non-isentropic Euler equations with solid wall boundary conditions. Submitted (2004).

[2] D. Bresch and B. Desjardins, Existence of global weak solutions for a 2D viscous shallow water equations and convergence to the quasi-geostrophic model. Comm. Math. Phys. 238 (2003) 211-223.

[3] D. Bresch, B. Desjardins and D. Gérard-Varet, Rotating fluids in a cylinder. Discrete Contin. Dynam. Systems Ser. A 11 (2004) 47-82.

[4] D. Bresch, B. Desjardins and C.-K. Lin, On some compressible fluid models: Korteweg, lubrication and shallow water systems. Comm. Partial Differential Equations 28 (2003) 1009-1037.

[5] D. Bresch, B. Desjardins, E. Grenier and C.-K. Lin, Low Mach number limit of viscous polytropic flows: formal asymptotics in the periodic case. Stud. Appl. Math. 109 (2002) 125-148.

[6] R. Danchin, Fluides légèrement compressibles et limite incompressible. Séminaire École Polytechnique (France), Exposé No. III (2000).

[7] B. Desjardins, E. Grenier, P.-L. Lions and N. Masmoudi, Incompressible limit for solutions of the isentropic Navier-Stokes equations with Dirichlet boundary conditions. J. Math. Pures Appl. 78 (1999) 461-471.

[8] I. Gallagher, Résultats récents sur la limite incompressible. Séminaire Bourbaki (France), No. 926 (2003). 
[9] J.F. Gerbeau and B. Perthame, Derivation of viscous Saint-Venant system for laminar Shallow water; Numerical results. Discrete Contin. Dynam. Systems Ser. B 1 (2001) 89-102.

[10] E. Grenier, Oscillatory perturbations of the Navier-Stokes equations. J. Math. Pures Appl. 76 (1997) 477-498.

[11] C.D. Levermore and M. Sammartino, A shallow water model with eddy viscosity for basins with varying bottom topography. Nonlinearity 14 (2001) 1493-1515.

[12] C.D. Levermore, M. Oliver and E.S. Titi, Global well-posedness for a models of shallow water in a basin with a varying bottom. Indiana Univ. Math. J. 45 (1996) 479-510.

[13] P.-L. Lions, Mathematical topics in fluid dynamics, Vol. 2, Compressible models. Oxford Science Publication, Oxford (1998).

[14] P.-L. Lions and N. Masmoudi, Incompressible limit for a viscous compressible fluids. J. Math. Pures Appl. 77 (1998) 585-627.

[15] G. Métivier and S. Schochet, The incompressible limit of the non-isentropic Euler equations. Arch. Rational Mech. Anal. 158 (2001) 61-90.

[16] G. Métivier and S. Schochet, The incompressible limit of the non-isentropic Euler equations, in Séminaire Équations aux Dérivées Partielles, École Polytechnique (2001).

[17] M. Oliver, Justification of the shallow water limit for a rigid lid with bottom topography. Theor. Comp. Fluid Dyn. 9 (1997) $311-324$.

[18] J. Pedlosky, Geophysical fluid dynamics. Berlin Heidelberg-New York, Springer-Verlag (1987).

To access this journal online: www.edpsciences.org 